\title{
Comparaison de populations de zébu malgache à l'aide des distances génétiques
}

\author{
P Souvenir Zafindrajaona, JJ Lauvergne \\ Institut national de la recherche agronomique, laboratoire de Génétique factorielle, \\ 78352 Jouy-en-Josas cedex, France
}

(Reçu le 14 octobre 1991, accepté le 22 avril 1993)

\begin{abstract}
Résumé - Au total, 1103 bovins malgaches des 2 sexes en provenance de 3 zones d'échantillonnage (nord-ouest, sud-ouest et centre) distantes en moyenne de $800 \mathrm{~km}$ ont été examinés pour les caractères visibles et 526 échantillons sanguins individuels ont été analysés. Il s'agissait de comparer le polymorphisme de gènes à effet visible, de gènes de groupes sanguins et de lactoprotéines dans 5 sous-populations représentatives de l'ensemble de l'île. Les résultats font tout d'abord apparaître une forte ressemblance entre les 2 grandes zones naisseuses (nord-ouest et sud-ouest) pour la variabilité génétique visible (indice de diversité de $\mathrm{Nei}=0.40$ ), ce qui implique que l'on est partout en présence d'une population traditionnelle. Les fréquences élevées du variant $A$ au locus kappa-Cn, de l'ordre de 0,70 , du variant $B$ au locus bêta- $L g$, de l'ordre de 0,68 et la morphologie du chromosome Y (acrocentrique) confirment l'appartenance au type zébu vrai. Les distances génétiques calculées montrent que les 5 sous-populations considérées semblent être génétiquement proches. Cela confirme l'impression d'homogénéité qui se dégageait déjà d'une précédente étude de distances génétiques biométriques.
\end{abstract}

zébu malgache / gène à effet visible / polymorphisme biochimique / distance génétique

Summary - Comparison of Malagasy zebu populations using genetic distances. $A$ total of 1103 Malagasy cattle of both sexes from 3 sampling regions (northwest, southwest and centre) over a distance of $800 \mathrm{~km}$ have been examined for visible traits and 526 blood samples have been analyzed. The aim was to compare the polymorphism of genes with visible effect, blood group loci and lactoprotein loci for 5 subpopulations representative of the whole island. The results show that the 2 large zones of birth have very similar visible genetic variability (Nei's diversity index $=0.40$ ) which indicates that everywhere the population is of traditional type. The high frequencies of the A variant (0.70) of the 
kappa-Cn locus, the B variant (0.68) of the beta-Lg locus as well as the morphology of the chromosome $Y$ (acrocentric) confirm that this Zebu population is of the true Zebu type. The analysis shows that the 5 subpopulations seem to be genetically close. This confirms the homogeneity already described in a previous study of biometrical distances.

malagasy zebu / gene with visible effect / biochemical polymorphism / genetic distance

\section{INTRODUCTION}

Dans un précédent article, nous avons constaté une grande ressemblance entre des populations de zébu malgache de zones distantes de $800 \mathrm{~km}$ pour leurs caractéristiques biométriques (Lauvergne et Souvenir, 1992). Ces mêmes échantillons ont été retenus, avec une autre zone du centre (une partie des Hauts-Plateaux et certaines régions de Sakay) pour une étude concernant cette fois la variabilité visible et le polymorphisme biochimique.

Cette homogénéité dans le format s'accompagne d'une grande variabilité pour certains caractères visibles conditonnés par des variants à des loci déjà identifiés (en particulier ceux de couleur du pelage). Cette variabilité est l'indication que l'on serait en présence d'une population de type traditionnel, au sens donné à ce terme par Lauvergne (1982) pour définir une catégorie taxonomique au sein des espèces animales domestiques.

Si le zébu malgache a déjà fait l'objet d'un début de comparaison avec les taurins pour le polymorphisme génétique des lactoprotéines (Grosclaude et al, 1974), en revanche aucune étude à partir des données de polymorphisme des loci à effet visible n'a encore été mise en œuvre.

Dans le présent article, on se propose d'utiliser 5 mesures de distances génétiques, à partir de certains loci à effet visible et de certains loci de polymorphisme biochimique, afin de mettre en évidence, de mesurer et de comparer le degré de «traditionalité» des populations bovines de 3 zones géographiques de l'île.

\section{MATÉRIEL ET MÉTHODES}

\section{Matériel}

Deux zones d'échantillonnage ont été choisies en fonction de leur forte densité bovine et de leur spécialisation en zone naisseuse : la zone 1 (nord-ouest) : population 1 , subdivisée en 2 sous-populations, et la zone 2 (sud-ouest) : population 2, subdivisée elle aussi en 2 sous-populations. Une troisième zone (centre) : population 3 , comportant un mélange d'animaux des 2 zones précédentes, a été également considérée dans notre étude.

De cette manière, les 5 sous-populations retenues constituent un réseau d'échantillonnage représentatif de l'ensemble de la population bovine malgache. La nonprise en compte de l'est de l'île est justifiée par le fait qu'il s'agit d'une zone uniquement utilisatrice. 


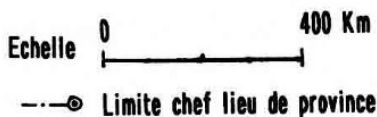

.$- \rightarrow$ Limite chef lieu de province

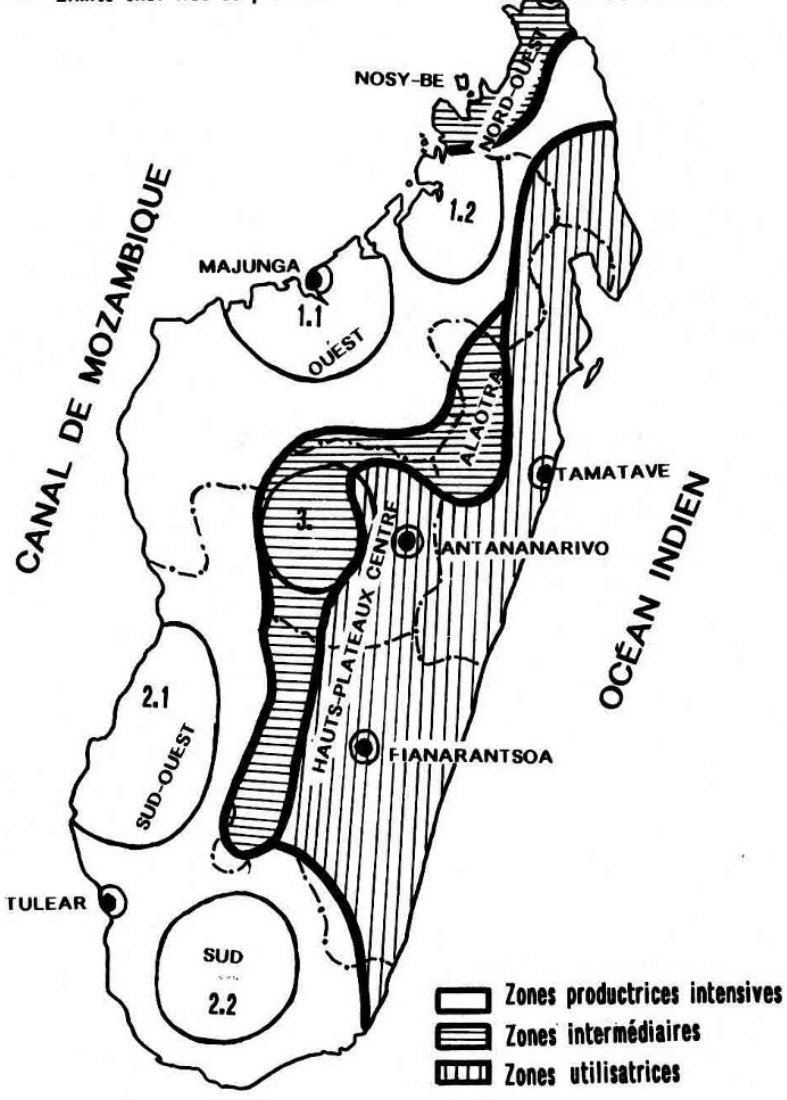

Fig 1. Délimitation des zones d'échantillonnage.

La délimitation des zones d'échantillonnage est donnée sur la figure 1, où l'on peut voir que les centres de gravité des zones prises 2 à 2 sont en moyenne éloignés de $800 \mathrm{~km}$.

Au total, 1103 animaux adultes ont été examinés pour les caractères visibles (tableau Ia) et 558 prélèvements sanguins, dont 368 pour les groupes sanguins, 180 pour les lactoprotéines, parmi lesquels 154 échantillons ont pu être analysés pour les génotypes du locus kappa-Cn et du locus bêta-Lg et 4 pour l'examen du chromosome Y (tableau Ib). 
Tableau I. Données recueillies sur le terrain. a. Profil visible. b. Prélèvements sanguins. a

\begin{tabular}{|c|c|c|c|c|c|c|c|}
\hline \multirow[t]{3}{*}{ Échantillonnage } & \multicolumn{6}{|c|}{ Animaux examinés } & \multirow{3}{*}{ Zone 3} \\
\hline & \multicolumn{3}{|c|}{ Zone 1} & \multicolumn{3}{|c|}{ Zone 2} & \\
\hline & 1.1 & 1.2 & Total & 2.1 & 2.2 & Total & \\
\hline Mâles & 96 & 83 & 179 & 104 & 89 & 193 & 20 \\
\hline Femelles & 131 & 134 & 265 & 161 & 124 & 285 & 161 \\
\hline Total & 227 & 217 & 444 & 265 & 213 & 478 & 181 \\
\hline
\end{tabular}

b

\begin{tabular}{cccc}
\hline Échantillonnage & Groupes sanguins & Lactoprotéines & Chromosome $Y$ \\
\hline 1.1 & 108 & 63 & 2 \\
1.2 & 102 & & \\
2.1 & 63 & 40 & 2 \\
2.2 & 47 & 51 & \\
3 & 48 & 154 & 4 \\
\hline
\end{tabular}

\section{Méthodes}

\section{Variations phénotypiques visibles}

\section{Traits visibles}

C'est l'ensemble des caractères morphologiques autres que la coloration :

- le profil céphalique (3 phénotypes);

- le port de l'oreille (3 phénotypes);

- le type de cornage ( 3 phénotypes);

- la position de la bosse ( 2 phénotypes).

D'après ce que l'on sait, ces caractères ne sont en général pas influencés par le milieu. Même si l'état de l'animal modifie le volume de la bosse, celle-ci ne change pas de place pour autant.

\section{Variants de coloration}

Quatre «dimensions» indépendantes de coloration de la robe proposées par Lauvergne (1983) ont été retenues pour cette étude :

- le patron pigmentaire (combinant l'eumélanine et la phæomélanine);

- le type d'eumélanine (noire ou brune); 
- l'altération pigmentaire (mélange intime de poils blancs et colorés);

- les panachures blanches (caractérisées par la forme des taches blanches et leur répartition).

En l'absence de connaissances suffisamment précises des rapports de dominance (en particulier au locus Agouti, $A$ ) et de pénétrance (pour tous les loci), on a considéré seulement les fréquences phénotypiques.

\section{Polymorphismes biochimiques}

Les échantillons de sang prélevés ont été analysés au laboratoire des groupes sanguins et au laboratoire de génétique biochimique de l'INRA à Jouy-en-Josas.

L'analyse du polymorphisme génétique des 11 systèmes de groupes sanguins ( $A, B, C, F, J, L, M, S, Z, R^{\prime}, T^{\prime}$ ) a été faite selon la technique de réaction d'hémolyse donnée en détail par Grosclaude et al (1979).

Le système $C$ est réduit à un ensemble tétra-allélique, par regroupement des phénogroupes comportant respectivement les facteurs antigéniques $C_{1}, C_{2}, C_{1}^{\prime \prime}$ et $C_{2}^{\prime \prime}$, dont les déterminants génétiques sont très proches, voire allèles, selon la carte établie par Guérin et al (1981).

En revanche, le système $B$, pour lequel il nous a été impossible d'identifier les phénogroupes (car on ne connaissait pas les ascendants du sujet considéré) et le système $M$, qui s'est avéré toujours monomorphe, n'ont pas été retenus dans notre analyse.

Pour identifier les génotypes des animaux aux loci kappa-Cn et bêta- $L g$, on a tout d'abord procédé à une amplification de l'ADN par la méthode PCR (polymerase chain reaction). Ensuite, les techniques d'analyse ont été celles de Vosberg (1989) développées par Levéziel et al (1990) pour la caséine kappa et de Medrano et Aguilar-Cordova (1990) pour la lactoglobuline bêta.

Les 11 systèmes retenus sont des systèmes génétiquement indépendants (Larsen, 1971), l'ensemble allélique ainsi défini compte au total 28 allèles (tableau II).

Tableau II. Ensemble allélique des 11 systèmes génétiques retenus.

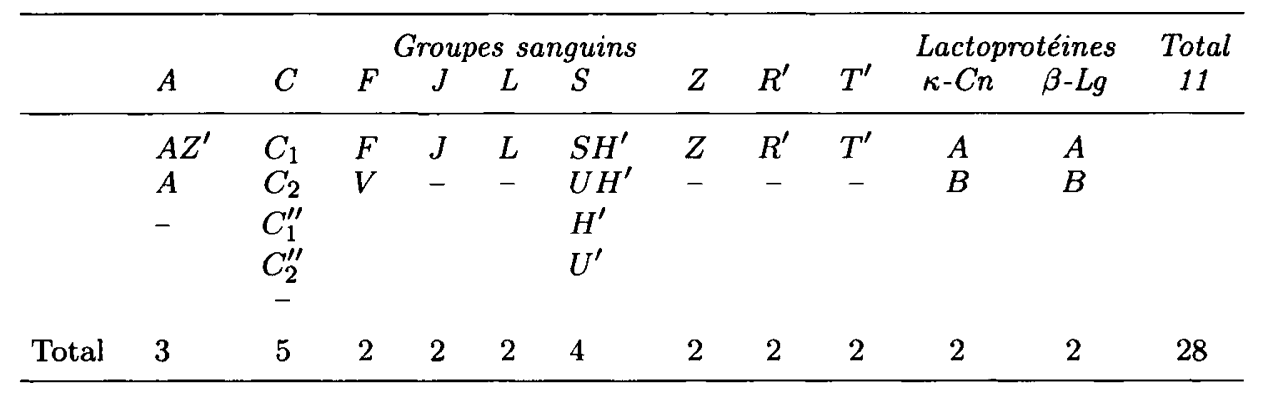

\section{Examen du caryotype}

L'identification du chromosome $\mathrm{Y}$ a été faite au laboratoire de cytogénétique de l'INRA à partir de cultures de lymphocytes avec une coloration au Giemsa. 


\section{Estimation des fréquences alléliques et mesures de la variabilité génétique}

L'estimation des fréquences alléliques par chaque méthode repose sur l'hypothèse d'équilibre selon la loi de Hardy-Weinberg, hypothèse qui a été testée sur des systèmes génétiques où les génotypes sont connus.

\section{Détermination des fréquences alléliques}

Pour les systèmes génétiques où les allèles sont codominants, les fréquences alléliques ont été calculées par comptage direct (Bouquet et Grosclaude, 1968), l'écarttype de l'erreur de la fréquence $q$ d'un allèle étant $\sigma=[q \times(1-q) / 2 N]^{1 / 2}$.

La méthode de la racine carrée a été utilisée pour les systèmes bi- ou trialléliques avec dominance. La fréquence $q$ d'un allèle récessif est estimée par la racine carrée de la fréquence observée des homozygotes récessifs, l'écart type de l'erreur étant approximativement $\sigma=[(1-\mathrm{q}) 4 \mathrm{~N}]^{1 / 2}$.

La méthode itérative proposée est beaucoup plus longue à décrire, la démarche est explicitée mathématiquement par Ceppellini et al (1956). Elle a été appliquée aux systèmes complexes $(A, C$ et $S)$.

\section{Taux d'hétérozygotie}

Le taux d'hétérozygotie a été mesuré par l'indice de diversité de Nei (1978) selon la formule :

$$
h_{k}=1-\sum_{i=1}^{i=l} p_{i}^{2}
$$

où $h$ représente la probabilité de tirer au hasard un individu hétérozygote au locus $k$ dans l'hypothèse de panmixie, $p_{i}$ étant la fréquence du $i$ allèle au locus, $l$ étant le nombre d'allèles.

Le taux d'hétérozygotie moyen est, quant à lui, la moyenne non pondérée des indices pour $n$ loci considérés :

$$
\bar{h}=1 / n \sum_{k=1}^{k=n} h_{k}
$$

En toute rigueur, la comparaison de populations par l'indice de diversité de Nei qui mesure le taux d'hétérozygotie doit être basée sur des groupes de loci de même effectif et ayant le même nombre d'allèles. Ainsi, avons-nous pris en considération d'une part les 5 loci de coloration, et de l'autre les 11 systèmes de polymorphisme biochimique pour lesquels les connaissances génétiques sont suffisantes.

Cet indice peut être utilisé de 2 façons :

- pour la mesure brute de la variabilité, en considérant les loci sur lesquels la sélection intentionnelle ne se manifeste pas (polymorphismes biochimiques);

- pour la mesure du degré de traditionalité basée sur la variabilité des loci de coloration qui sont polymorphes. 


\section{Distances génétiques}

Cinq formules différentes ont été utilisées pour mesurer la distance génétique entre 2 populations à partir des fréquences alléliques. Dans toutes ces formules de distance, $r$ est le nombre de loci considérés, $m_{j}$ le nombre d'allèles au j ${ }^{\mathrm{e}}$ locus et $x_{i j}$ et $y_{i j}$ les fréquences du $i^{e}$ allèle au $j^{e}$ locus dans les populations $X$ et $Y$.

La distance de Cavalli-Sforza (1969)

$$
f_{\theta}=\left[4 \sum_{j=1}^{r}\left(1-\sum_{i=1}^{m_{j}}\left(x_{i j} \cdot y_{i j}\right)^{1 / 2}\right] / \sum_{j=1}^{r}\left(m_{j}-1\right)\right.
$$

La distance de Rogers (1972)

$$
D_{R}=\sum_{j=1}^{r}\left[\sum_{i=1}^{m_{j}}\left(x_{i j}-y_{i j}\right)^{2}\right] / 2 r
$$

La distance absolue de Gregorius (1984)

$$
d_{0}=1 / 2 r \sum_{j=1}^{r} \cdot \sum_{i=1}^{m_{j}}\left|x_{i j}-y_{i j}\right|
$$

La distance standard de Nei (1972)

$$
D_{N}=-\log _{e} I
$$

avec $I=J_{X Y} /\left(J_{X} \cdot J_{Y}\right)^{1 / 2}$

$$
J_{X}=1 / r \sum_{j=1}^{r} \cdot \sum_{i=1}^{m_{j}} x_{i j}^{2} ; \quad J_{Y}=1 / r \sum_{j=1}^{r} \cdot \sum_{i=1}^{m_{j}} y_{i j}^{2} ; J_{X Y}=1 / r \sum_{j=1}^{r} \cdot \sum_{i=1}^{m_{j}} x_{i j} \cdot y_{i j}
$$

La distance minimale de Nei (1972)

$$
D_{m}=1 / 2 r \sum_{j=1}^{r} \cdot \sum_{i=1}^{m_{j}}\left(x_{i j}-y_{i j}\right)^{2}
$$

Des méthodes d'analyse avec des représentations graphiques ont été également utilisées pour interpréter les différentes matrices de distances calculées, comme le dendrogramme, par la méthode UPGMA de Sneath et Sokal (1973) ou l'analyse en coordonnées principales de la méthode de Lefebvre (1976 et 1983), pour préciser les proximités génétiques existant entre les différentes sous-populations. 


\section{RÉSULTATS}

\section{Variabilité interne des populations}

\section{Variation visible}

Pour cette analyse, seules ont été considérées les populations des zones d'échantillonnage 1 et 2 . La zone 3 (centre) n'a pas été retenue sachant qu'on y rencontre un mélange d'animaux en provenance des 2 zones précédentes.

\section{Traits visibles}

L'examen comparatif des phénotypes visibles analysés révèle une grande uniformité des animaux au sein des populations des 2 zones d'échantillonnage. On observe en effet que la variation phénotypique est absente pour 3 caractères sur $4:$ le profil de la tête, le port de l'oreille et la position de la bosse (tableau III).

En ce qui concerne le type de cornage, les cornes en lyre (mâles : resp $25,14 \%$ et $32,12 \%$, femelles : resp $24,80 \%$ et $30,53 \%$ pour les populations 1 et 2 ) et en croissant (mâles : resp $55,86 \%$ et $66,32 \%$, femelles : resp $58,80 \%$ et $67,72 \%$ ) prédominent chez le zébu malgache adulte, avec les mêmes proportions de type de cornage entre les mâles et les femelles pour les populations considérées. Le type de cornage en coupe, qui est un type à cornes courtes, semble être une forme transitoire entre les cornages en lyre et en croissant. En effet, ce type de cornes courtes ne représente à partir de l'âge de 5 ans qu'un pourcentage très faible, de l'ordre de $10 \%$ (tableau III).

Tableau III. Profils phénotypiques visibles (traits morphologiques identifiés) chez les zébus malgaches.

\begin{tabular}{|c|c|c|c|c|c|c|c|c|c|}
\hline \multirow[t]{3}{*}{ Caractères } & \multirow[t]{3}{*}{ Phénotypes } & \multicolumn{4}{|c|}{ Zone 1} & \multicolumn{4}{|c|}{ Zone 2} \\
\hline & & \multicolumn{2}{|c|}{ Mâles } & \multicolumn{2}{|c|}{ Femelles } & \multicolumn{2}{|c|}{ Mâles } & \multicolumn{2}{|c|}{ Femelles } \\
\hline & & $n$ & $\%$ & $n$ & $\%$ & $n$ & $\%$ & $n$ & $\%$ \\
\hline Type de tête & Rectiligne & 179 & 100 & 265 & 100 & 193 & 100 & 285 & 100 \\
\hline Port de l'oreille & Horizontal & 167 & 100 & 249 & 100 & 181 & 100 & 273 & 100 \\
\hline \multirow[t]{3}{*}{ Type de cornage } & Coupe & 34 & 19 & 43 & 16,40 & 3 & 1,56 & 5 & 1,75 \\
\hline & Lyre & 45 & 25,14 & 65 & 24,80 & 62 & 32,12 & 87 & 30,53 \\
\hline & Croissant & 100 & 55,86 & 154 & 58,80 & 128 & 66,32 & 193 & 67,72 \\
\hline $\begin{array}{l}\text { Position } \\
\text { de la bosse }\end{array}$ & Thoraco-thoracique & 179 & 100 & 265 & 100 & 193 & 100 & 285 & 100 \\
\hline
\end{tabular}

\section{Variants de coloration}

Cinq loci de coloration sont identifiables chez les zébus malgaches (tableau IV) : $A$ (Agouti) avec 5 allèles, $D$ (Dilution) avec 2 allèles, $S$ (Spotting) avec 2 allèles, $B l$ (Blason) avec 2 allèles et $C s$ (Color-sided) avec 2 allèles selon la terminologie de Lauvergne (1983). 
Tableau IV. Liste des gènes de coloration identifiés chez les zébus malgaches, nomenclature proposée par Lauvergne (1983).

\begin{tabular}{lccc}
\hline \multirow{2}{*}{ Nom } & Loci & Nom & Allèles \\
& Symbole & self & $A^{s}$ \\
Agouti & $A$ & tan & $A^{t}$ \\
& & blackish & $A^{b}$ \\
& & red & $A^{r}$ \\
& & light extremities & $A^{l}$ \\
Roan & $R n$ & wild & $R n^{+}$ \\
& & roan & $R n^{r}$ \\
Blason & $B l$ & wild & $B l^{+}$ \\
& & hereford & $B l^{h}$ \\
Color-sided & $C s$ & wild & $C s^{+}$ \\
& & telemark & $C s^{t}$ \\
Spotting & $S$ & wild & $S^{+}$ \\
& & piebald & $S^{p}$ \\
\hline
\end{tabular}

Presque toutes les couleurs et les patrons connus sont présents dans les 2 populations, ce qui corrobore bien l'hypothèse d'appartenance à une population traditionnelle où ségrègent encore de nombreux variants à effet visible.

On note ensuite que les fréquences phénotypiques sont semblables d'une zone à l'autre.

Il faut également remarquer une prédominance du patron eumélanique (avec des fréquences de 0,285 à 0,335 ) parmi les patrons pigmentaires et une prédominance du phénotype flancs colorés chez les mâles $(0,230$ et 0,310 pour, resp, les populations 1 et 2$)$ et celui de blason chez les femelles $(0,210$ et 0,266$)$ pour la panachure blanche. Tous les autres patrons et panachures sont présents à des fréquences très faibles (tableau V).

\section{Polymorphismes biochimiques et sériques}

\section{Fréquences alléliques}

L'hypothèse de panmixie a été testée pour les loci de Caséine kappa, de lactoglobuline bêta et du système $F$, à partir des fréquences génotypiques obtenues par comptage direct (tableau VI). Le test de $\chi^{2}$ pour les sous-populations n'a pas révélé de différence significative à $5 \%$ pour la sous-population 1 . En revanche, dans les sous-populations 2 et 3 , la loi n'est pas vérifiée dans respectivement 2 cas (caséine kappa et lactoglobuline bêta) et 1 cas (lactoglobuline bêta). Cet écart, qui est dû sans doute à la faiblesse des effectifs, ne devrait pas remettre en cause notre hypothèse générale de panmixie, que l'on peut admettre pour les autres systèmes.

$\mathrm{Si}$, ensuite, on considère les résultats du tableau VII, on voit que :

- pour certains systèmes les fréquences alléliques varient dans de faibles proportions, comme celle de l'allèle $M$ du système $M$ toujours égale à 0 et celle de l'allèle $R^{\prime}$ du système $R^{\prime}$, qui est toujours inférieure à 0,04 ; 


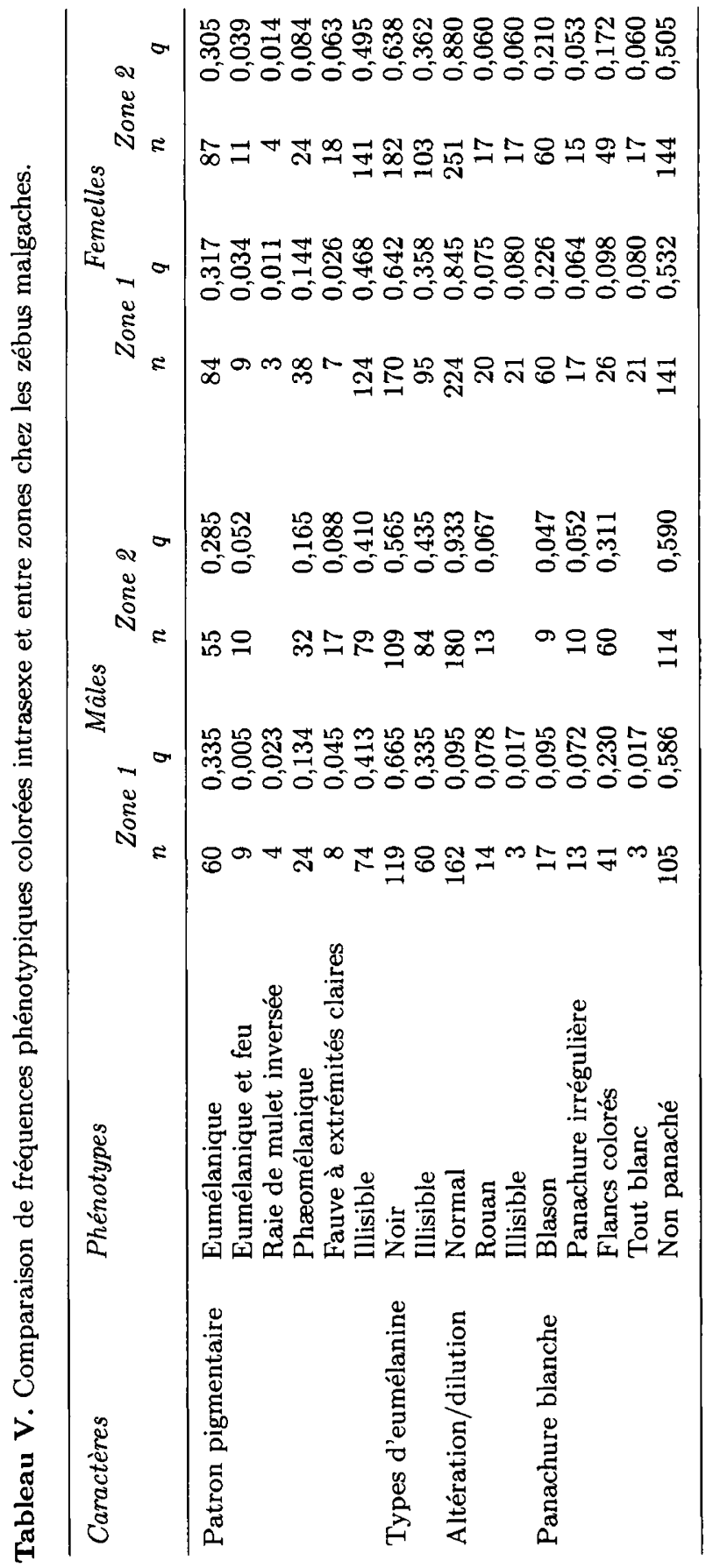


Tableau VI. Test de l'équilibre génétique (loi de Hardy-Weinberg).

\begin{tabular}{|c|c|c|c|c|c|c|}
\hline \multirow[t]{2}{*}{ Sous-population } & \multirow[t]{2}{*}{ Locus } & \multicolumn{4}{|c|}{ Effectifs observés } & \multirow[t]{2}{*}{ Valeur de $\chi^{2}$} \\
\hline & & $\begin{array}{c}A A \\
(F F)\end{array}$ & $\begin{array}{c}\tilde{A B} \\
(F V)\end{array}$ & $\begin{array}{c}B B \\
(V V)\end{array}$ & Total & \\
\hline \multirow{3}{*}{1} & $\kappa-\mathrm{Cn}$ & 18 & 33 & 12 & 63 & $0,20 \mathrm{~ns}$ \\
\hline & $\beta$-Lg & 8 & 32 & 23 & 63 & $0,18 \mathrm{~ns}$ \\
\hline & F & 174 & 36 & 00 & 210 & $1,78 \mathrm{~ns}$ \\
\hline \multirow{3}{*}{2} & $\kappa-\mathrm{Cn}$ & 16 & 24 & 00 & 40 & $7,34^{* *}$ \\
\hline & $\beta-\operatorname{Lg}$ & 6 & 9 & 25 & 40 & $7,12^{* *}$ \\
\hline & $\mathrm{F}$ & 82 & 28 & 00 & 110 & $2,34 \mathrm{~ns}$ \\
\hline \multirow{3}{*}{3} & $\kappa-\mathrm{Cn}$ & 28 & 19 & 4 & 51 & $0,09 \mathrm{~ns}$ \\
\hline & $\beta-\mathrm{Lg}$ & 8 & 15 & 28 & 51 & $4,70^{*}$ \\
\hline & $F^{\circ}$ & 33 & 15 & 00 & 48 & $1,64 \mathrm{~ns}$ \\
\hline
\end{tabular}

ns : non significatif à $P<0,05 ;^{*}$ significatif à $P<0,05 ;^{* *}$ : significatif à $P<0,01$; $\mathrm{ddl}=1$.

- pour d'autres systèmes, les variations des fréquences alléliques semblent réparties de façon identique, comme pour l'allèle $F(0,86-0,91)$ avec un écart type de même ordre de grandeur $(\sigma=0,03)$ du système $F$, l'allèle $J(0,25-0,29$ et $\sigma=0,05)$ du système $J$ et l'allèle $T^{\prime}(0,85-0,90$ et $\sigma=0,03)$ du système $T^{\prime}$, qui font apparaître des points communs entre les populations;

- en revanche, il est des systèmes pour lesquels les variations des fréquences sont plus importantes et aident à évaluer la mesure de proximité génétique des populations, comme celles des allèles $A(0,11-0,30$ et $\sigma=0,06)$ du système $A, C_{1}^{\prime \prime}(0,37-0,52)$ et $C_{2}^{\prime \prime}(0,17-0,29)$ du système $C, L(0,41-0,57)$ du système $L, H^{\prime}(0,36-0,47)$ du système $S$ et $Z(0,27-0,58)$ du système $Z$.

Le polymorphisme aux loci de caséine kappa (kappa-Cn) et de lactoglobuline bêta (bêta- $L g$ ) permet l'identification des variants universellement répandus chez les bovins kappa-Cn $A$ et kappa-Cn $B$, bêta- $L g A$ et bêta- $L g B$. Les fréquences géniques sont données dans le tableau VIII.

On note la fréquence élevée du variant $A$ de kappa- $C n$, de l'ordre de 0,70 , avec un écart type du même ordre de grandeur, égal à 0,04 , et celle du variant $B$ du bêta- $L g$ de l'ordre de 0,68 , avec un écart-type aussi égal à 0,04 .

Un test d'homogénéité appliqué aux fréquences calculées dans les 3 zones a été effectué (tableau IX).

\section{Taux d'hétérozygotie (indice de diversité de Nei)}

Les résultats présentés dans le tableau $\mathrm{X}$ montrent que, quelle que soit l'origine des données (fréquences des allèles aux loci de coloration ou fréquences des allèles de groupes sanguins ou groupes sanguins et lactoprotéines), les indices de diversité moyens étaient du même ordre et très élevés dans les 2 zones naisseuses (resp 0,45 ; 0,$45 ; 0,41$ et 0,$44 ; 0,44 ; 0,41$ ). Les valeurs pour la population 3 (centre) différaient quelque peu $(0,39)$ mais on sait qu'il s'agit d'un mélange d'animaux en provenance des 2 zones précédentes. 
Tableau VII. Fréquences alléliques des systèmes de groupes sanguins.

\begin{tabular}{|c|c|c|c|c|c|c|}
\hline \multirow[b]{2}{*}{ Systèmes } & \multirow[b]{2}{*}{ Allèles } & \multicolumn{5}{|c|}{ Sous-populations } \\
\hline & & 1.1 & 1.2 & 2.1 & 2.2 & 3 \\
\hline \multirow[t]{2}{*}{$A$} & $\begin{array}{c}A \\
A Z^{\prime}\end{array}$ & $\begin{array}{l}0,300 \\
0,005\end{array}$ & $\begin{array}{l}0,197 \\
0.005\end{array}$ & 0,213 & 0,113 & 0,278 \\
\hline & - & $\begin{array}{l}0,005 \\
0,695\end{array}$ & 0,798 & 0,787 & 0,007 & 0,722 \\
\hline \multirow[t]{4}{*}{$C$} & $C_{1}$ & 0,090 & 0,112 & 0,193 & 0,102 & 0,155 \\
\hline & $C_{2}$ & 0,225 & 0,226 & 0,156 & 0,196 & 0,095 \\
\hline & $C_{1}^{\prime \prime}$ & 0,517 & 0,376 & 0,382 & 0,408 & 0,610 \\
\hline & $C_{2}^{\prime \prime}$ & 0,168 & $\begin{array}{l}0,256 \\
0,030\end{array}$ & 0,269 & 0,294 & 0,140 \\
\hline \multirow[t]{2}{*}{$F$} & $F$ & 0,917 & 0,912 & 0,880 & 0,862 & 0,844 \\
\hline & $V$ & 0,083 & 0,088 & 0,120 & 0,138 & 0,156 \\
\hline \multirow[t]{2}{*}{$J$} & $J$ & 0,267 & 0,293 & 0,255 & 0,285 & 0,290 \\
\hline & - & 0,733 & 0,707 & 0,745 & 0,715 & 0,710 \\
\hline \multirow[t]{2}{*}{$L$} & $L$ & 0,570 & 0,568 & 0,410 & 0,474 & 0,323 \\
\hline & - & 0,430 & 0,432 & 0,590 & 0,526 & 0,677 \\
\hline$M$ & - & 1,000 & 1,000 & 1,000 & 1,000 & 1,000 \\
\hline \multirow[t]{4}{*}{$S$} & $S H^{\prime}$ & 0,214 & 0,154 & 0,156 & 0,168 & 0,233 \\
\hline & $U H^{\prime}$ & 0,138 & 0,176 & 0,204 & 0,134 & 0,025 \\
\hline & $H^{\prime}$ & 0,368 & 0,410 & 0,366 & 0,470 & 0,465 \\
\hline & $U^{\prime}$ & 0,280 & 0,260 & 0,274 & 0,228 & 0,277 \\
\hline \multirow[t]{2}{*}{$Z$} & $Z$ & 0,580 & 0,380 & 0,288 & 0,270 & 0,250 \\
\hline & - & 0,420 & 0,620 & 0,712 & 0,730 & 0,750 \\
\hline \multirow[t]{2}{*}{$R^{\prime}$} & $R^{\prime}$ & 0,043 & 0,030 & 0,008 & 0,022 & 0,200 \\
\hline & - & 0,957 & 0,970 & 0,002 & 0,978 & 0,800 \\
\hline \multirow[t]{2}{*}{$T^{\prime}$} & $T^{\prime}$ & 0,904 & 0,860 & 0,875 & 0,854 & 0,677 \\
\hline & - & 0,096 & 0,140 & 0,125 & 0,146 & 0,323 \\
\hline
\end{tabular}

Tableau VIII. Fréquences du variant $A$ de la caséine kappa et du variant $B$ de la lactoglobuline bêta.

\begin{tabular}{lcc}
\hline Zones d'échantillonnage & $\kappa-C n A$ & $\beta-L g B$ \\
\hline Nord-nord-ouest & 0,55 & 0,63 \\
Sud-sud-ouest & 0,70 & 0,74 \\
Centre & 0,74 & 0,70 \\
Madagascar* & 0,75 & 0,73 \\
\hline
\end{tabular}

* Résultats obtenus par Grosclaude et al (1974).

Morphologie du chromosome $Y$

Sur tous les échantillons de mâles analysés, on observait un chromosome Y petit et acrocentrique (fig 2). 
Tableau IX. Test d'homogénéité appliqué aux fréquences calculées dans les populations des 3 zones d'échantillonnage.

\begin{tabular}{lcccc}
\hline Variants & Nord/nord-ouest & Sud/sud-ouest & Centre & $\chi^{2}$ \\
\hline \multirow{3}{*}{$\kappa-C n A$} & 0,55 & 0,70 & 0,74 & \\
& 18 & 16 & 28 & $0,720 \mathrm{~ns}$ \\
& $(19,05)$ & $(19,60)$ & $(27,90)$ & \\
$\beta-L g B$ & 0,63 & 0,74 & 0,70 & \\
& 24 & 25 & 28 & $0,840 \mathrm{~ns}$ \\
& $(25)$ & $(21,90)$ & $(24,99)$ & \\
\hline
\end{tabular}

Entre parenthèses, les effectifs théoriques; ns : non significatif à $P<0,05$.

Tableau X. Taux d'hétérozygotie moyen (indice de diversité de Nei) par groupe de loci et par zone d'échantillonnage.

\begin{tabular}{lcccccccc}
\hline Groupe de loci & \multicolumn{3}{c}{ Nord/nord-ouest } & \multicolumn{3}{c}{ Sud/sud-ouest } & \multicolumn{2}{c}{ Centre } \\
& 1.1 & 1.2 & 1 & 2.1 & 2.2 & 2 & 3 \\
\hline Coloration & 0,42 & 0,48 & 0,45 & 0,41 & 0,46 & 0,44 & 0,39 \\
$\begin{array}{l}\text { Groupes sanguins } \\
\text { Groupes sanguins } \\
\quad \\
\begin{array}{c}+ \\
\text { lactoprotéines }\end{array}\end{array}$ & 0,36 & 0,36 & 0,45 & 0,36 & 0,34 & 0,44 & 0,39 \\
& & & & & & & \\
\hline
\end{tabular}

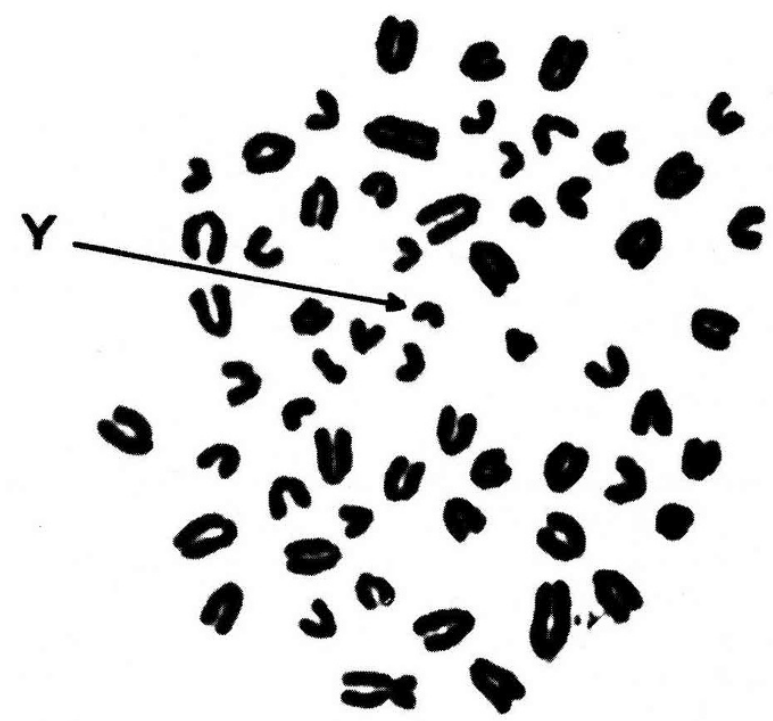

Fig 2. Examen du caryotype (identification du chromosome $\mathrm{Y}$ ). 


\section{Variabilité entre populations}

\section{Distances génétiques}

Les estimations établies au moyen des 5 formules différentes selon le groupe de loci pris en compte présentent des résultats similaires, avec des coefficients de corrélation supérieurs à 0,90 (tableaux XIa et $\mathrm{b}$ ).

Tableau XI. Les matrices de coefficients de corrélation des distances génétiques. a. Groupe des loci de coloration. b. Groupe des loci des groupes sanguins.

a

\begin{tabular}{lcccc}
\hline & $f_{\theta}$ & $D_{R}$ & $d_{0}$ & $D_{m}$ \\
\hline$D_{R}$ & 0,94 & & & \\
$d_{0}$ & 0,93 & 0,99 & & \\
$D_{m}$ & 0,93 & 0,90 & 0,90 & \\
$D_{N}$ & 0,92 & 0,90 & 0,90 & 0,99 \\
\hline
\end{tabular}

b

\begin{tabular}{lcccc}
\hline & $f_{\theta}$ & $D_{R}$ & $d_{0}$ & $D_{m}$ \\
\hline$D_{R}$ & 0,96 & & & \\
$d_{0}$ & 0,96 & 0,99 & & \\
$D_{m}$ & 0,95 & 0,96 & 0,95 & \\
$D_{N}$ & 0,95 & 0,95 & 0,95 & 0,99
\end{tabular}

Toutefois, les matrices de distances sont difficilement interprétables sans l'utilisation de représentation graphiques. C'est ainsi que nous avons donné les représentations par les dendogrammes et par l'analyse en coordonnées principales.

\section{Dendogrammes}

Les résultats sont présentés sur la figure 3 (a et b), selon le groupe de loci pris en compte

On constate que les variations de la structure des dendogrammes sont peu nombreuses et ne portent que sur les sous-populations 1.2 de la zone ouest, 2.1 de la zone sud et 3 de la zone centre.

On observe également que, quels que soient la distance mesurée et le type de loci considérés (coloration ou groupes sanguins), les 5 sous-populations sont très proches les unes des autres.

\section{Analyse en coordonnées principales}

L'analyse en coordonnées principales (ACP) de Lefebvre (1976 et 1983) a été faite à partir des matrices de distance absolue de Gregorius (1984).

Avec cette méthode, les 5 sous-populations précédemment décrites peuvent être situées les unes par rapport aux autres (fig $4 \mathrm{a}$ et b).

On remarque que la population 3 qui représente la zone 3 (centre) occupe une superficie importante, et que l'incertitude sur la position relative des souspopulations 1.2 et 2.1 est levée : on peut les rattacher respectivement aux souspopulations 1.1 et 2.2 . 


\section{DISCUSSION}

\section{Classement en zébu vrai ou sanga}

La classification adoptée par des auteurs qui ont étudié les bovins sur le continent africain et à Madagascar, comme Payne (1970), tient compte en premier lieu de la présence/absence de bosse, pour distinguer les 2 sous-espèces Bos taurus (taurin) et Bos indicus (zébu). Plus récemment, la pertinence de ce classement a été renforcée par l'examen du chromosome $\mathrm{Y}$, petit et acrocentrique chez le zébu et submétacentrique chez le Taurin (Kieffer et Cartwright, 1968; Meyer, 1984).

L'identification de ce chromosome sexuel n'est pas seulement utile pour différencier les taurins des zébus vrais. Il s'avère en outre que ce polymorphisme de $Y$ semble avoir des effets nocifs dans les croisements zébu $\times$ taurin. Il a été en effet constaté que les produits entre une race de zébu (à $\mathrm{Y}$ acrocentrique) et une race taurine (à $Y$ submétacentrique) donnaient en $F_{2}$ un taux de vêlage significativement inférieur à celui des 2 races parentales (Rendel, 1980; Rao, 1982).

À leur tour, les zébus ont été classés par Epstein (1933) et Curson (1936) en zébus vrais (d'origine asiatique) et en sangas, quelquefois appelés pseudo-zébus (d'origine africaine), le critère de distinction étant la position de la bosse : thoracothoracique pour le zébu vrai, cervico-thoracique pour le sanga. Des auteurs comme Epstein $(1955,1956,1957)$ ou Payne $(1964,1991)$ pensent, quant à eux, que le sanga proviendrait d'un croisement entre zébu et taurin.

Dans cette perspective taxonomique, nos observations indiqueraient que les bovins autochtones à bosse de Madagascar devraient être rangés parmi les zébus vrais : ils présentaient tous une bosse thoraco-thoracique et tous les mâles dont le caryotype a été établi (fig 2) possédaient un chromosome Y petit et acrocentrique.

Cette classification est confirmée par la fréquence élevée des allèles de certains groupes sanguins érythrocytaires : $\mathrm{q}_{(-)}=1,0$ pour l'allèle négatif du système $M$, $\mathrm{q}_{(-)}$compris entre 0,95 et 0,99 pour l'allèle négatif du système $R^{\prime}$ et $\mathrm{q}_{(\mathrm{T})}$ entre 0,85 et 0,90 pour l'allèle $T$ du système $T^{\prime} ; \mathrm{q}_{(\mathrm{A})}$ de l'ordre de 0,70 pour le variant $A$ de caséine kappa, $\mathrm{q}_{(\mathrm{B})}$ de l'ordre de 0,68 pour le variant $B$ et la fréquence élevée du variant $A$ de lactoglobuline bêta. En effet, selon certains auteurs, ces fréquences constituent également un critère de discrimination entre les zébus de l'Inde, de l'Afrique et les taurins d'Europe (Aschaffenburg et al, 1968; Baker et Manwell, 1980 et 1991 ; Prasad et Nair, 1983).

En revanche, on peut signaler que l'allèle $A Z^{\prime}$ du système $A$, très courant chez les zébus mais absent dans les races de taurins d'Europe du Nord (Grosclaude $e$ t $a l, 1990)$, n'est observé chez le zébu malgache que dans la zone du nord-ouest, avec la fréquence très faible de 0,005 .

\section{Degré de «traditionalité»}

On a vu que les populations traditionnelles se distinguaient des races standardisées par l'accumulation de variants visibles en ségrégation, et qu'il était possible de mesurer ce degré de standardisation par l'indice de diversité de Nei, établi à partir de loci de coloration fixés dans les races standardisées, en ségrégation dans les populations traditionnnelles (tableau X). 
$10(5 \cdot 5)$

$\mathrm{DR}(5 \cdot 5)$
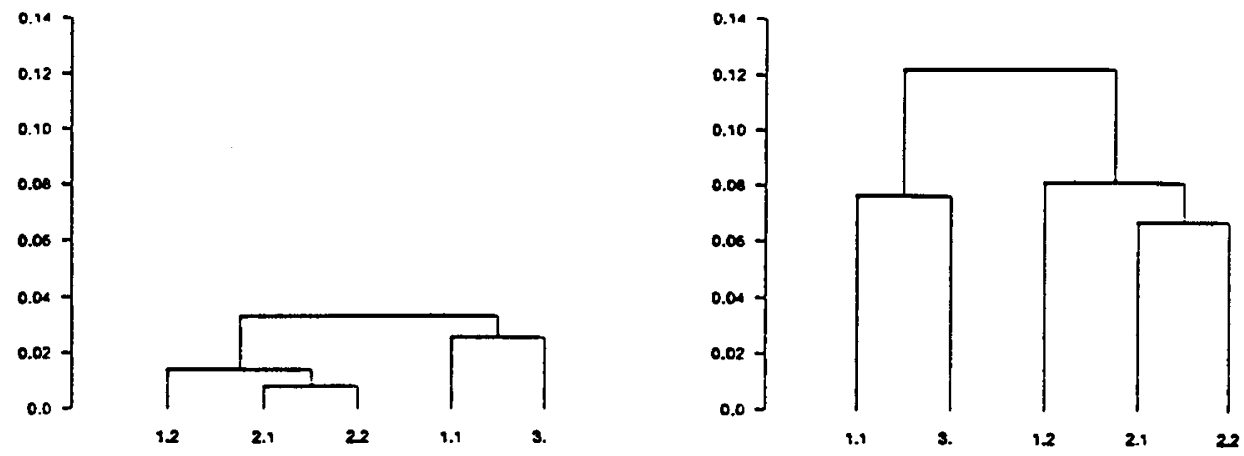

$D G(5-5)$

$\operatorname{Dm}(5-3)$
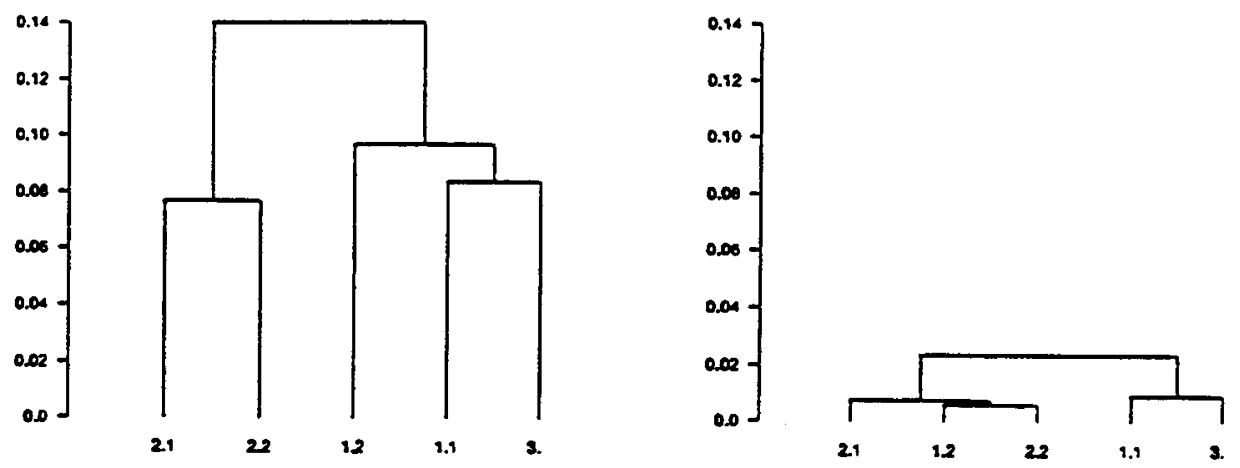

DN (5-5)

$\left.\begin{array}{l}0.16 \\ 0.12 \\ 0.10 \\ 0.08 \\ 0.08 \\ 0.04 \\ 0.02 \\ 0.0\end{array}\right]$

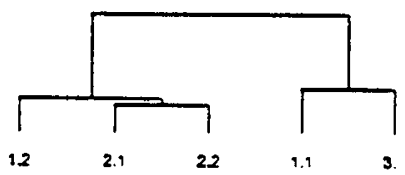

Fig 3. a. Représentation graphique par les dendrogrammes de distances génétiques calculées à partir des loci de coloration. 
$f(5-9)$

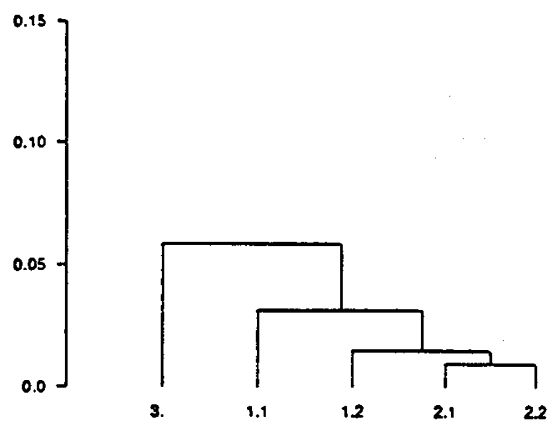

$D G(5 \cdot 9)$

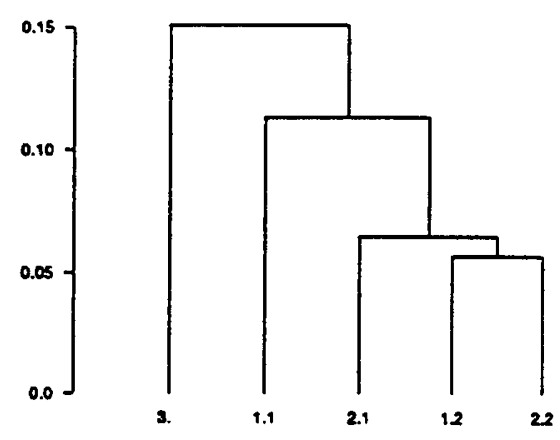

$\operatorname{DN}(5 \cdot 9)$
$\operatorname{DR}(5 \cdot 9)$

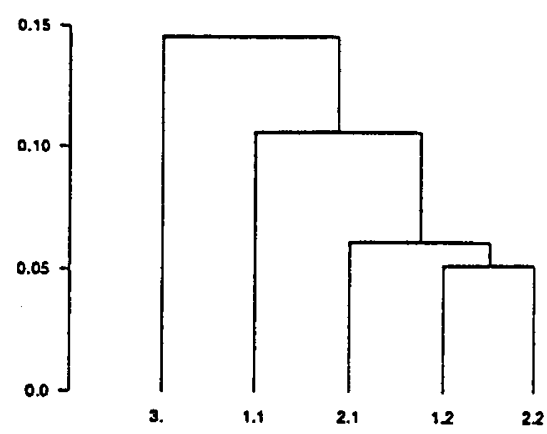

$\operatorname{Dm}(5 \cdot 9)$

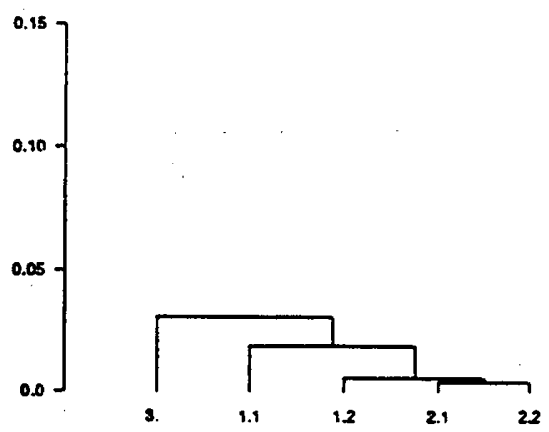

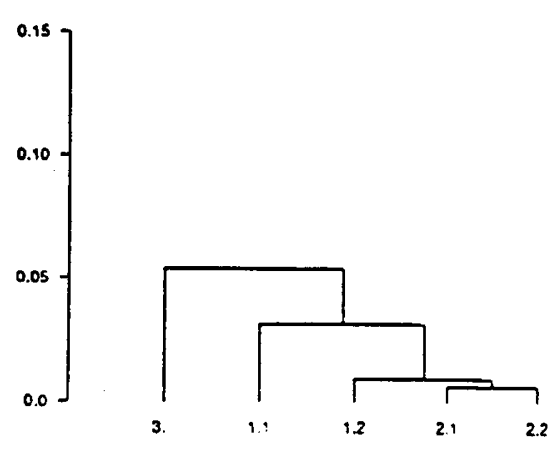

Fig 3. b. Représentation graphique par les dendrogrammes de distances génétiques calculées à partir de loci de groupes sanguins. 

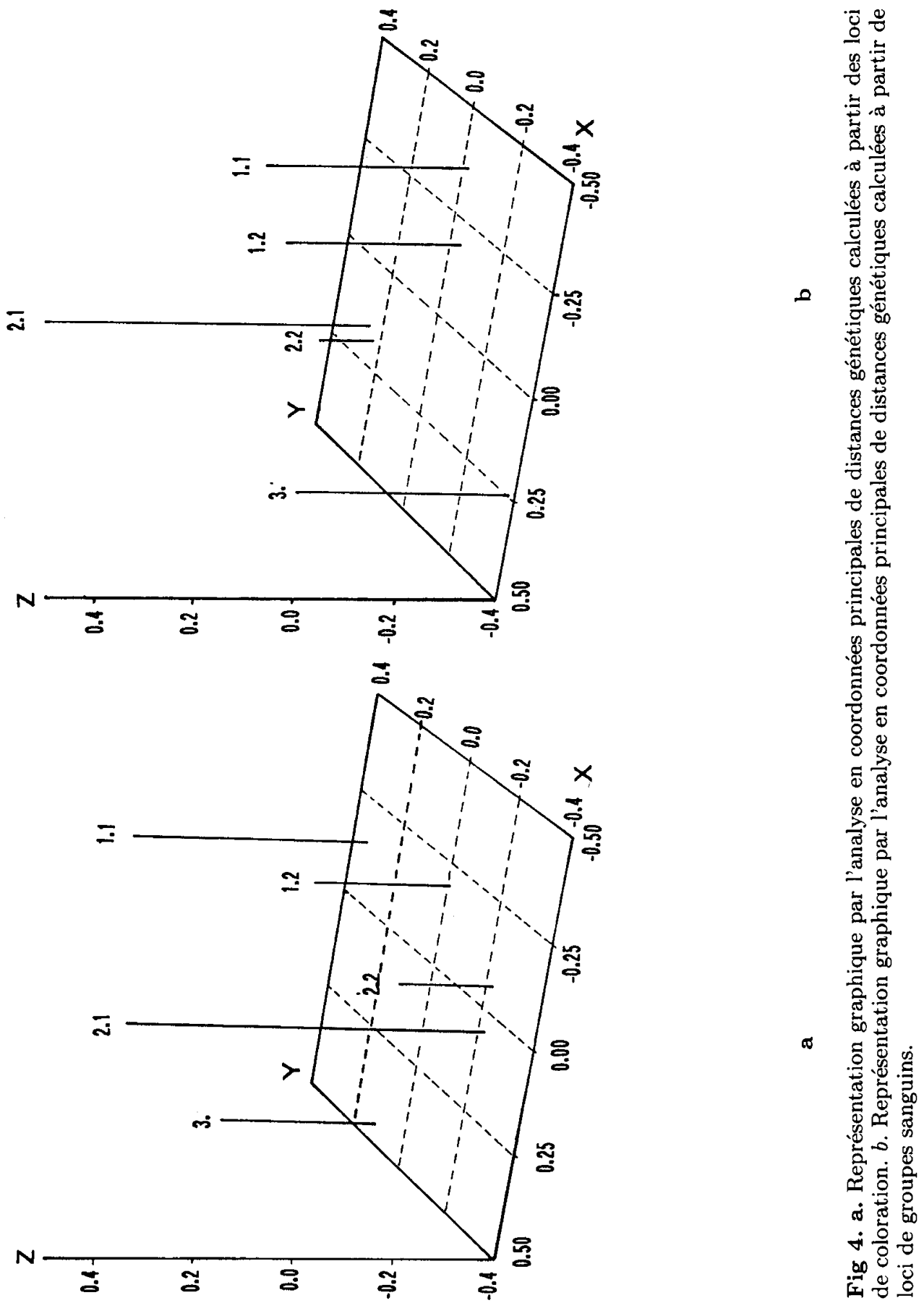
Dans notre étude, les indices de diversité moyens pour les loci de coloration prennent tous des valeurs élevées et voisines, comprises entre 0,39 et 0,45 , ce qui corrobore l'hypothèse d'appartenance à une population de type traditionnel. On observe cependant une certaine tendance à la standardisation, qui se manifeste par la fréquence élevée de certains phénotypes (patron pigmentaire eumélanique noir et dessin flancs colorés). Cette dérive pourrait être due à des préférences rituelles. On sait par exemple que, lors des funérailles, on aime sacrifier des mâles adultes de grande taille, uniformément noirs ou à défaut, rouges avec des cornes, qui se dirigent vers le ciel (Decary, 1951).

Pour les bovins, malheureusement, on ne dispose pas encore d'un corpus de mesures de la diversité génétique aux loci de coloration, qui nous permettrait de faire des comparaisons avec les résultats que nous avons obtenus sur le zébu malgache. En revanche chez la chèvre, une étude des populations traditionnelles du rivage nord de la Méditerranée a indiqué que ces indices étaient de 0,07 pour une population substandardisée et de 0,41 pour une population traditionnelle (Lauvergne et al, 1988).

À Madagascar les valeurs pour le zébu correspondent ainsi assez bien aux estimations données pour les populations traditionnelles de chèvres.

Ainsi pourrait-on proposer, pour la mesure de la «traditionalité» d'une population domestique, un «degré de traditionalité» égal à l'indice de diversité de Nei appliqué aux loci de coloration.

\section{Degré d'homogénéité}

\section{Distances génétiques}

On observe tout d'abord que toutes les formules utilisées donnent des résultats similaires. En outre, les variations de fréquences alléliques observées dans chaque population ne semblent pas modifier de façon notable les estimations de distance, comme on l'a vu dans le tableau IX, où les variations ne sont pas significatives pour la fréquence du variant $A$ du locus $K-C n$ et celle du variant $B$ du locus $\beta-L g$, qui indiquent que la population de zébu malgache présente une réelle homogénéité.

\section{Représentation en dendrogrammes}

D'une manière générale, l'examen des dendrogrammes montre que les divergences entre les populations sont faibles (fig $3 \mathrm{a}$ et b).

\section{Groupe de loci de coloration}

On constate que les variations sont peu nombreuses entre certains dendrogrammes, ce qui montre que, quelle que soit la méthode utilisée pour mesurer les distances, les 5 sous-populations semblent former un seul groupe variant seulement dans sa structure interne. 
Groupe de loci des groupes sanguins

En considérant les dendrogrammes obtenus par les fréquences des groupes sanguins, on observe que seules les sous-populations 1.2 et 2.1 présentent une incertitude sur leur position. Quoi qu'il en soit, la structure du groupe ne semble pas perturbée par cette incertitude.

\section{Représentation par l'analyse en coordonnées principales}

On voit apparaître un rattachement en quelque sorte attendu : la sous-population 1.2 confirme sa proximité avec la 1.1 au sein de la zone nord et la sous-population 2.1 avec la 2.2 au sein de la zone sud.Ainsi peut-on mieux situer des sous-populations dont la position restait incertaine, à l'exception de la population 3 qui, il est vrai, est constituée par des apports en provenance des 2 zones de l'ouest et du sud.

Ainsi, au niveau même de la variabilité génétique, se confirme une ressemblance entre les zones nord et sud.

\section{Degré de variabilité}

Pour les indices de diversité de Nei calculés à partir des loci de polymorphismes biochimiques sanguins, on observe que les valeurs obtenues dans la population traditionnelle de zébu malgache sont fort proches de celles calculées par Aupetit (1985) pour une série de races standardisées d'Europe (tableau X).

Comme Epstein (1971) a fait remarquer que le foyer d'origine des zébus se trouve en Asie du Sud-Ouest et que les bovins qui sont à l'origine des races africaines seraient aussi, en partie, à l'origine des races européennes, alors il pourrait s'agir sans doute d'une diversité des polymorphismes communs à l'espèce bovine.

On note en outre, comme précédemment, que les valeurs des indices de diversité de Nei calculés pour les loci visibles sont très proches des valeurs prises par les indices calculés pour les loci de polymorphisme biochimique.

Comme les indices de diversité de Nei calculés à partir des polymorphismes biochimiques sont les mêmes dans toutes les catégories (races standardisées ou populations traditionnelles), il n'y a en quelque sorte que l'indice de diversité à partir des gènes à effet visible qui permette de caractériser les populations traditionnelles.

\section{CONCLUSIONS}

Après avoir mis en évidence dans un premier article (Lauvergne et Souvenir, 1992) que la population bovine malgache était composée de zébus vrais dont les caractéristiques biométriques sont assez constantes d'une zone de l'île à l'autre, la présente étude nous permet de conclure que la population de zébus malgaches est de type traditionnel (d'après l'importante variabilité visible observée). Les critères d'évaluation étant fort semblables d'une zone à l'autre, cela vient confirmer notre première impression d'homogénéité des ressources génétiques bovines dans toute l'île. 
Finalement, le seul critère permettant de déceler une variation utile pour l'explication des origines resterait l'indice auriculaire, comme cela a été mis en évidence dans le précédent article (Lauvergne et Souvenir, 1992).

Toutefois, on ne dispose pas actuellement des données de ce type sur le continent africain.

\section{REMERCIEMENTS}

Nous exprimons notre gratitude aux techniciens de l'élevage du CRZ de Kianjasoa et du CRZ de Miadana, qui nous ont aidés à la collecte des données à Madagascar, aux différentes équipes du laboratoire des groupes sanguins dirigé par D François, du laboratoire de génétique biochimique dirigé par $\mathrm{P}$ Martin et du laboratoire de cytogénétique dirigé par M Popescu, pour leur coopération efficace. Ces laboratoires appartiennent au département de génétique animale de l'INRA et sont basés au centre de recherche de Jouy-en-Josas.

Nous remercions également $\mathbf{J}$ Lefebvre pour son assistance dans les calculs informatiques.

\section{RÉFÉRENCES}

Aschaffenburg RA, Sen A, Thompson MP (1968) Genetic variants of casein in Indian and African zebu cattle. Comp Biochem Physiol 25, 177-184

Aupetit RY (1985) Analyse des relations phylogénétiques entre les races bovines françaises par le polymorphisme biochimique. Thèse de doctorat, $3^{\mathbf{e}}$ cycle, université Paris VII, $66 \mathrm{p}$

Baker CMA, Manwell C (1991) Population genetics, molecular markers and gene conservation of bovine breeds. In : Cattle genetic resources (CG Hickman, ed). Elsevier, Amsterdam, 221-304

Bouquet Y, Grosclaude F (1968) Groupes sanguins et situation génétique de la race bovine flamande. Ann Biol Anim Biochem Biophys 8, 463-483

Cavalli-Sforza LL (1969) Human diversity. In : Proc 12th Intern Cong Genet Tokyo, 1968, 3, 405-416

Ceppellini R, Siniscalco M, Smith CAB (1956) The estimation of gene frequencies in a random-mating population. Ann Hum Genet 20, 97-115

Curson HH (1936) A comparison of two of the so-called Zebus. J Vet Sci Anim 7, 371-391

Decary R (1951) Mœurs et coutumes des Malgaches. Payot, Paris, $280 \mathrm{p}$

Epstein H (1933) Descent and origin of the Afrikander cattle. J Hered 24, 448-462

Epstein H (1955) The zebu cattle of East Africa. Bast Afr Agric J 21, 83

Epstein H (1956) The origin of Afrikander cattle with comments on the classification and evolution of zebu cattle in general. Z Tierz ZüchtBiol 66, 97-148

Epstein H (1957) The Sanga of East Africa. Afr Agric J 22, 149-164

Epstein H (1971) The origin of domestic animals of Africa. Africana Publishing Corporation, New York, 2 vol, 573-719 p

Gregorius HR (1984) A unique genetic distance. Biom J 26, 13-18

Grosclaude F, Mahé MF, Mercier JC (1974) Comparaison du polymorphisme génétique des lactoprotéines du zébu et des bovins. Ann Génét Sél Anim 6, 305329 
Grosclaude F, Guérin G, Houlier G (1979) The genetic map of the $B$ system of cattle blood groups as observed in French breeds. Anim Blood Groups. Biochem Genet 10, 199-212

Grosclaude F, Aupetit RY, Lefebvre J, Mériaux JC (1990) Essai d'analyse des relations génétiques entre les races bovines françaises à l'aide du polymorphisme biochimique. Genet Sel Evol 22, 317-338

Guérin G, Grosclaude F, Houlier G (1981) The $C$ system of cattle blood groups. 2. Partial genetic map of the system. Anim Blood Groups Biochem Genet 12, 171-191 Kieffer NM, Cartwright TC (1968) Sex chromosome polymorphism in domestic cattle. $J$ Hered 59, 35-36

Larsen B (1971) Blood groups and polymorphic proteins in cattle and swine. Ann Génét Anim 3, 59-70

Lauvergne JJ (1982) Génétique des populations animales après la domestication : conséquences pour la conservation des races. 2nd World Congr Genet Appl Livestock Prod, Madrid, 1982, 6, 77-87

Lauvergne JJ (1983) Les robes du bœuf domestique (Bos taurus) et leur formule génétique. Ethnozoot 32, 70-90

Lauvergne JJ (1988) Méthodologie proposée pour l'étude des Ovicaprinœ méditerranéens en 1986. In : Populations traditionnelles et premières races standardisées $d$ Ovicaprinæ dans le bassin méditerranéen (JJ Lauvergne, ed). Coll INRA ${ }^{\circ} 47$, INRA, Paris, 77-94

Lauvergne JJ, Renieri C, Pieramati C (1988) Le scénario de peuplement caprin méditerranéen ancien. In : Populations traditionnelles et premières races standardisées d'Ovicaprinæ dans le bassin méditerranéen (JJ Lauvergne, ed). Coll INRA, $\mathrm{n}^{\circ}$ 47, INRA, Paris, 253-265

Lauvergne JJ, Souvenir Zafindrajaona $P$ (1992) Comparaison de 2 populations de zébus malgaches par les distances génétiques biométriques. Rev Élev Méd Vét Pays Trop 45, 167-174

Lefebvre J (1983) Introduction aux analyses statistiques multidimensionnelles (exemples d'application). Masson, Paris, $274 \mathrm{p}$

Levéziel H, Diamini G, Martin P, Pilla F, Caccio S, Sgaramella V (1990) Characterization of the bovine casein genes by the polymerase chain reaction; XXII Int Conf Anim Genet East-Lansing, 23-31 August 1990, Abstracts 2.1.4

Manwell C, Baker CMA (1980) Chemical classification of cattle. 2. Phylogenetic tree and specific status of zebu. Anim Blood Groups Biochem Genet 11, 151-162

Medrano JF, Aguilar Cordova E (1990) Polymerase chain reaction amplification of bovine beta-lactoglobulin genomic sequences and identification of genetic variants by RFLP analysis. Anim Biotech 1, 73-77

Meyer EHH (1984) Classification of Bos indicus cattle breeds in southern and central Africa as Sanga or zebu type by means of chromosome morphology. 6th Eur Coll Cytogenet Domest Anim Zurich, 1984, 96-103

Nei M (1972) Genetic distance between populations. Am Naturalist 106, 283-292

Nei M (1978) Estimation of average heterozygosity and genetic distance from small number of individuals. Genetics $89,583-592$

Payne WJA (1964) The origin of domestication of cattle in Africa. Emp J Exp Agric $32,97-113$ 
Payne WJA (1970) Cattle production in the tropics. 1. Breeds and breeding. Longman, London, 62-70

Payne WJA (1991) Domestication : a forwards step in civilization. In : Cattle genetic resources (CG Hickman, ed). Elsevier, Amsterdam, 51-72

Prasad SK, Nair PG (1983) Immunogenetic profile of erythrocyte antigens in cattle. Part II. Genetic architecture of complex blood groups of Indian cattle. Indian J Exp Biol 21, 168-172

Rao AVN (1982) Causes and incidence of reproductive disorders among zebu $\times$ taurus crossbreed cows in Andhra Pradesh. Theriogenology 17, 189-191

Rendel JM (1980) Low calving rate in Brahman cross cattle. Theor Appl Genet 58, 207-210

Rogers JS (1972) Measures of genetic similarity and genetic distance. Studies in Genetics VII. University of Texas, publ no 7213, 145-153

Sneath PHA, Sokal RR (1973) Numerical taxonomy. Freeman, San Francisco

Vosberg MP (1989) The polymerase chain reaction, an improved method for the analysis of nucleic acids. Hum Genet 83, 1-15 